\title{
Age- and gender-related differences in the evolution of psychological and cognitive status after the lockdown for the COVID-19 outbreak: a follow-up study
}

\author{
Gianpaolo Maggi ${ }^{1}$ - Ivana Baldassarre ${ }^{1}$ Andrea Barbaro ${ }^{1}$. Nicola Davide Cavallo ${ }^{1} \cdot$ Maria Cropano ${ }^{1}$. \\ Raffaele Nappo ${ }^{1} \cdot$ Gabriella Santangelo $^{1}$ (])
}

Received: 10 October 2021 / Accepted: 16 November 2021 / Published online: 25 November 2021

(c) Fondazione Società Italiana di Neurologia 2021

\begin{abstract}
Objective Individuals experienced psychological symptoms in response to quarantine for the COVID-19 pandemic. Therefore, we aimed to investigate the possible effect of age and gender on the evolution of mental health status after the quarantine in the Italian population and the baseline predictors of post-traumatic stress symptoms.

Methods An online follow-up questionnaire including an assessment of depression, anxiety, anger, post-traumatic stress, subjective cognitive failures, resilience, and coping style was completed by participants 2 months after the end of the quarantine $(n=758)$.

Results Individuals experienced psychological symptoms also 2 months after the end of the quarantine. No decrease in depression and anxiety scores emerged, but younger individuals and females experienced more severe symptoms. Anger symptoms decreased in young adults, whereas they increased in older adults. Moreover, individuals reported more cognitive failures at follow-up. No changes were observed in resilience, whereas participants reported adopting fewer coping strategies at follow-up. Finally, post-traumatic stress symptoms 2 months after the end of the lockdown were associated with more severe psychological symptoms and more fear of getting infected at baseline.

Conclusions Our findings demonstrate that the long-term psychological impact and the cognitive consequences of quarantine differ according to age and gender. The identification of more vulnerable groups allows the implementation of interventions to reduce psychological symptoms and the risk for cognitive impairment.
\end{abstract}

Keywords COVID-19 $\cdot$ Mental Health · Cognitive difficulties $\cdot$ Longitudinal changes $\cdot$ Young adults · Gender

\section{Introduction}

A novel coronavirus (SARS-CoV-2) is responsible for an infectious disease commonly defined as coronavirus disease 2019 (COVID-19) which causes a severe respiratory illness.

The population density together with multimorbidity, advanced age, and male gender represent the main risk factors associated with the spread of contagion and the mortality rate [1], but the consequent measures implemented to control the spread of COVID-19 such as lockdowns and self-isolation have heavily impacted on individuals' mental

Gabriella Santangelo

gabriella.santangelo@unicampania.it

1 Department of Psychology, University of Campania "Luigi Vanvitelli”, Viale Ellittico 31, 81100 Caserta, Italy health [2]. In fact, people complained more depressive symptoms, anxiety, stress, and post-traumatic stress (PTS) symptoms in parallel with the spread of the virus [3]. Moreover, psychological symptoms due to quarantine for COVID19 have been related to more severe cognitive failures [4] but also to the development of PTS disorder [5]; therefore, the long-term consequences of psychological symptoms deserve to be further investigated.

Some studies investigated possible differences in severity or frequency of psychological disturbances due to gender or age. Women are more at risk of psychological disturbances than men [6], whereas mixed results have been reported by analyzing different age groups. In fact, some studies reported that young adults experienced more psychological effects from COVID-19 pandemic despite appearing to be less vulnerable to the most serious medical complications associated with COVID-19 [7-9]; conversely, other studies suggested to 
pay more attention to older adults' mental health since they need to practice social distancing limiting their interactions also with family members due to their frailty and vulnerability to COVID-19 [10, 11].

Furthermore, although the immediate psychological response to the outbreak and quarantine was widely investigated, few studies investigated the long-term psychological consequences of COVID-19 pandemic and self-isolation measures. In China, Zhou and collaborators [12] recruited participants from Wuhan before and after the lockdown lifting and showed that a majority of people continued to implement self-isolation and reported a slight improvement of the psychological well-being after the end of the lockdown. In Europe, Daly and colleagues [13] revealed a pronounced and prolonged deterioration in mental health occurred due to outbreak of COVID-19 pandemic in the United Kingdom (UK).

In Italy, previous longitudinal studies $[14,15]$ explored the psychological changes in the Italian population during different phases of the lockdown period. Roma and colleagues [14] revealed an increase in stress and depression over the course of the lockdown; change in stress and depression was not influenced by gender, whereas an increase in stress level was associated with younger age. Furthermore, Salfi and collaborators [15] showed that women reported higher scores than men on sleep and mental health scales at the beginning of the lockdown, but they exhibited a slight trend toward improvement of mental health in the following weeks, unlike male participants who reported a substantial increment of perceived stress.

However, these studies did not explore whether age- and gender-related differences in psychological effects of the quarantine (i.e., depression, anxiety, perceived stress) were stable or changed after many months from the end of the lockdown.

Moreover, the prevalence of PTS symptoms in the Italian population ranged from 20 to $29 \%$ during the quarantine $[5,16]$ and higher levels of stress perceived during the restrictive measures period were found to be associated with female gender, younger age, lower levels of education, and the exposition to stressful situations (e.g., having to go out to work, having an acquaintance infected with COVID-19) [16, 17]. However, until now, no study investigated the baseline predictors of PTS symptoms after the end of the quarantine.

Therefore, we performed a follow-up study in order to explore (i) the possible differences in the evolution of cognitive and psychological status due to age and gender and (ii) the baseline predictors of possible post-traumatic stress in the Italian general population.

The present study could facilitate the recognition of more vulnerable groups to specific psychological and cognitive symptoms in order to implement personalized interventions according to different age and gender groups.

\section{Materials and methods}

\section{Participants}

The baseline study [4] included 4175 respondents who completed an online questionnaire employed on the virtual platform of Google Moduli to assess the psychological consequences of quarantine and lockdown measures due to the COVID-19 pandemic. Out of these, 2524 agreed to be contacted for the follow-up evaluation providing their email address. However, 59 respondents supplied an invalid email address, 18 reported an email address that did not match the one previously given, and 6 emails could not be delivered due to the full mailbox. Therefore, the final sample of the present study consisted of 758 respondents who were invited to complete the online survey described previously [4], but with the addition of a questionnaire assessing stress. Participation in the present survey was open from July 20 to October 7, 2 months after the end of quarantine and lockdown measures implemented by the Italian government.

The present study was approved by the Local Ethics Committee and conformed to the principles embodied in the Declaration of Helsinki. Individuals read and agreed to a digital informed consent to participate in the present study.

\section{Survey description}

The structured questionnaire consisted of several sections: informed consent; sociodemographic data (i.e., age, gender, employment status); mental health status (i.e., depression, anxiety, anger); personal resilience; coping style; subjective cognitive failures; and stress. The stress section was added in the follow-up study to evaluate stress due to quarantine/self-isolation for COVID-19.

The references of the tools employed in the present survey are reported in Supplemental Material 1.

\section{Sociodemographic data}

In the first survey [4], sociodemographic data such as gender, age, education level, residential location, marital and living status, household size, and employment status were collected. Moreover, respondents were asked to indicate the number of days spent in quarantine/self-isolation, housing characteristics, indirect or direct contact with an individual with COVID-19, and previous psychiatric illnesses; respondents were also asked to rate the frequency of feeling boredom, frustration, and fear of getting infected with COVID-19. 
In the follow-up survey, participants were asked about their employment status after the quarantine and to rate again the frequency of boredom, frustration, and fear of getting infected feelings.

\section{Mental health status}

Detailed description and scoring of tools employed in the survey are provided in Supplemental Material 1.

Depressive symptoms were evaluated using the Italian version of the Patient Health Questionnaire-9 (PHQ-9), a self-report 9-item inventory exploring symptoms of a major depressive episode as defined by the DSM-5.

Anxiety symptoms were assessed using the 7-item Generalized Anxiety Disorder scale (GAD-7) evaluating the DSMIV symptoms for generalized anxiety disorder.

Anger was assessed using the DSM-5 Level 2-AngerAdult measure, a 5-item version of the PROMIS Anger Short-Form assessing the severity of an individual's anger during the past 7 days (DSM-5-Anger).

Lastly, we added one statement "Have you ever had a fit of tears" to assess further depressive symptomatology. This item was rated on a 4-point Likert scale ranging from 0 "not at all" to 3 "nearly every day."

\section{Cognitive failures}

Cognitive failures were evaluated in the first and the followup survey in order to explore the long-term cognitive consequences of the quarantine.

Cognitive failures consist of frequent difficulties reported by individuals in cognitive control and were assessed by the Perceived Memory and Attentional Failures Questionnaire (PerMAFaQ), developed in a previous study [4].

\section{Resilience}

Resilience was evaluated in the first and the follow-up survey by the Italian translation of the Brief Resilience Scale (BRS), assessing the response of individuals to stressful situations.

\section{Coping style}

Coping style was evaluated in the first and the follow-up survey by the Italian translation of the Coping Scale, a selfreport questionnaire consisting of 13 items.

\section{Post-traumatic stress symptoms}

The subjective distress caused by the restrictive limitations was evaluated in the follow-up study by the Italian version of the Impact of Event Scale-Revised (IES-R), a self-report 22-item scale comprising three subscales: intrusion ( 8 items), avoidance (8 items), and hyperarousal (6 items).

\section{Statistical analysis}

We calculated descriptive statistics for sociodemographic data, pandemic and quarantine/self-isolation information, and variables assessing mental health status, cognitive failures, personal resilience, and coping style of respondents.

To analyze differences in mental health status scores reported at baseline and follow-up evaluations, we carried out several $2 \times 2 \times 3$ mixed-design ANOVA with time (baseline, follow-up) as within-subject factors and age (young adults, middle-aged adults, older adults) and gender (male, female) as between-subject factors for each mental health score (i.e., PHQ-9, GAD-7, DSM-5-Anger, PerMAFaQ, BRS, and Coping Scale). Bonferroni-corrected post hoc comparisons were performed in case of significant results.

Finally, to explore possible predictors of PTS symptoms within the whole sample and within male and female participants, we carried out several simple linear regression analyses entering IES-R as dependent variable and variables about demographic data, characteristics related to the quarantine, and the mental health status at baseline as independent variables. Multiple regression analyses (by stepwise method) were performed to investigate the most influential predictors of PTS symptoms entering significant factors from simple regression analyses as independent variables. The significance level was set at $\alpha=0.05$, and all statistical analyses were performed using SPSS Statistic 26.0.

\section{Results}

\section{Respondents' characteristics}

The follow-up survey was completed by 758 subjects (Table 1). The majority of participants were women, aged from 18 to 30 years, with a high educational level. Most participants were employed during the quarantine/self-isolation, but the majority was in smart working; after the quarantine, most participants were back to their workplace whereas $7.3 \%$ lost the job.

\section{Mental health status evolution over time}

\section{Depression}

Analysis of depressive symptoms revealed no significant main effects of the time $\left(F_{(1,752)}=1.885 ; p=0.170\right.$; $\left.\eta_{p}^{2}=0.003\right)$ and gender $\left(F_{(1,752)}=3.458 ; p=0.063\right.$; $\left.\eta_{p}^{2}=0.005\right)$. There was a significant main effect of the age $\left(F_{(2,752)}=10.666 ; p<0.001 ; \eta_{p}^{2}=0.028\right)$. 
Table 1 Sociodemographic data of the sample

\section{Variables}

\begin{tabular}{|c|c|c|c|c|c|}
\hline \multirow[t]{2}{*}{ Age } & 18-30 (Young adults) & $\begin{array}{l}\text { 31-50 (Middle-aged } \\
\text { adults) }\end{array}$ & 51 or more (Older adults) & & \\
\hline & $398(52.5 \%)$ & $226(29.8 \%)$ & $134(17.7 \%)$ & & \\
\hline \multirow[t]{2}{*}{ Sex } & Female & Male & & & \\
\hline & $566(74.7 \%)$ & $192(25.3 \%)$ & & & \\
\hline \multirow[t]{2}{*}{ Level of education } & Elementary & Middle school & High school & $\begin{array}{l}\text { Degree and post- } \\
\text { degree }\end{array}$ & \\
\hline & $1(0.1 \%)$ & $22(2.9 \%)$ & $223(29.4 \%)$ & $512(67.6 \%)$ & \\
\hline \multirow[t]{2}{*}{ Marital status } & Married & Unmarried/maiden & Divorced/ separated & Widower & \\
\hline & $203(26.8 \%)$ & $511(67.4 \%)$ & $40(5.3 \%)$ & $4(0.5 \%)$ & \\
\hline \multirow{2}{*}{$\begin{array}{l}\text { Number of people per } \\
\text { household }\end{array}$} & Alone/1 & 2 & $3-5$ & 6 or more & \\
\hline & $80(10.6 \%)$ & $183(24.3 \%)$ & $468(62 \%)$ & $23(3.1 \%)$ & \\
\hline \multirow{2}{*}{$\begin{array}{l}\text { Number of children per } \\
\text { Household }\end{array}$} & 0 & $1-2$ & 3 or more & & \\
\hline & $613(81.5 \%)$ & $126(16.8 \%)$ & $13(1.7 \%)$ & & \\
\hline \multirow{2}{*}{$\begin{array}{l}\text { Number of rooms in a } \\
\text { house }\end{array}$} & $1-2$ & 3 & 4 & 5 or more & \\
\hline & $61(8.2 \%)$ & $130(17.4 \%)$ & $232(31 \%)$ & $326(43.4 \%)$ & \\
\hline \multirow[t]{2}{*}{ House with... } & 1 or more windows & $\begin{array}{l}\text { Outdoor space (terrace. } \\
\text { balcony. garden or } \\
\text { shared courtyard) }\end{array}$ & & & \\
\hline & $54(7.1 \%)$ & $704(92.9 \%)$ & & & \\
\hline \multirow{2}{*}{$\begin{array}{l}\text { Employment status } \\
\text { during the quarantine/ } \\
\text { self-isolation }\end{array}$} & Unemployed & Students & Employed & Retired & \\
\hline & $62(8.1 \%)$ & $241(31.8 \%)$ & $436(57.6 \%)$ & $19(2.5 \%)$ & \\
\hline \multirow{2}{*}{$\begin{array}{l}\text { Work modalities during } \\
\text { the quarantine/self- } \\
\text { isolation }\end{array}$} & Smart working & Office & No job & & \\
\hline & $253(33.4 \%)$ & $87(11.5 \%)$ & $418(55.1 \%)$ & & \\
\hline \multirow{2}{*}{$\begin{array}{l}\text { Employment status } \\
\text { changes after the } \\
\text { quarantine/self- } \\
\text { isolation }\end{array}$} & $\begin{array}{l}\text { No (still in smart work- } \\
\text { ing) }\end{array}$ & No (still in office) & No (still unemployed) & $\begin{array}{l}\text { Yes (back to work- } \\
\text { place) }\end{array}$ & Yes (lost the job) \\
\hline & $149(19.7 \%)$ & $45(5.9 \%)$ & $254(33.5 \%)$ & $255(33.6 \%)$ & $55(7.3 \%)$ \\
\hline \multirow[t]{2}{*}{ Healthcare workers } & Hospital staff & $\begin{array}{l}\text { Other (e.g., psycholo- } \\
\text { gists, laboratory tech- } \\
\text { nicians, or medical } \\
\text { waste handlers) }\end{array}$ & Non-medical workers & & \\
\hline & $37(5 \%)$ & $15(1.9 \%)$ & $706(93.1 \%)$ & & \\
\hline \multirow{2}{*}{$\begin{array}{l}\text { Duration of quarantine/ } \\
\text { self-isolation }\end{array}$} & Mean (SD) & Median & & & \\
\hline & 30.37 (10.93) & 30 & & & \\
\hline \multirow{2}{*}{$\begin{array}{l}\text { Number of times going } \\
\text { out in a week during } \\
\text { the quarantine }\end{array}$} & 0 & $1-2$ & $3-4$ & 5 or more & \\
\hline & $247(32.6 \%)$ & $387(51.1 \%)$ & $61(8 \%)$ & $63(8.3 \%)$ & \\
\hline \multirow[t]{2}{*}{$\begin{array}{l}\text { Being affected by } \\
\text { COVID-19 }\end{array}$} & No & Yes (1 asymptomatic) & I do not answer & $\begin{array}{l}\text { No (symptomatic but } \\
\text { not tested by swab) }\end{array}$ & Yes (remitted) \\
\hline & $741(97.8 \%)$ & $1(0.1 \%)$ & $7(0.9 \%)$ & $6(0.8 \%)$ & $3(0.4 \%)$ \\
\hline $\begin{array}{l}\text { Direct contact with } \\
\text { people affected by } \\
\text { COVID-19 }\end{array}$ & I do not answer & No & Yes & & \\
\hline
\end{tabular}


Table 1 (continued)

\begin{tabular}{|c|c|c|c|c|c|}
\hline \multicolumn{6}{|l|}{$\underline{\text { Variables }}$} \\
\hline & $7(0.9 \%)$ & $708(93.4 \%)$ & $43(5.7 \%)$ & & \\
\hline \multirow{2}{*}{$\begin{array}{l}\text { Indirect contact with } \\
\text { people affected by } \\
\text { COVID-19 }\end{array}$} & I do not answer & No & Yes & & \\
\hline & $3(0.4 \%)$ & $413(54.5 \%)$ & $342(45.1 \%)$ & & \\
\hline \multirow{2}{*}{$\begin{array}{l}\text { Diagnosis of psychopa- } \\
\text { thology }\end{array}$} & I do not answer & No & Yes & & \\
\hline & $10(1.3 \%)$ & $692(91.3 \%)$ & $56(7.4 \%)$ & & \\
\hline \multirow[t]{2}{*}{ Boredom } & Never & Sometimes & Often & Always & Mean (SD) \\
\hline & $279(36.8 \%)$ & $287(37.9 \%)$ & $141(18.6 \%)$ & $51(6.7 \%)$ & $1.95(0.91)$ \\
\hline \multirow[t]{2}{*}{ Frustration } & Never & Sometimes & Often & Always & Mean (SD) \\
\hline & $240(31.7 \%)$ & $274(36.1 \%)$ & $164(21.6 \%)$ & $80(10.6 \%)$ & $2.11(0.97)$ \\
\hline \multirow{2}{*}{$\begin{array}{l}\text { Fear of getting infected } \\
\text { with COVID-19 }\end{array}$} & Never & Sometimes & Often & Always & Mean (SD) \\
\hline & $181(23.9 \%)$ & $328(43.3 \%)$ & $188(24.8 \%)$ & $61(8 \%)$ & $2.17(0.88)$ \\
\hline \multirow[t]{2}{*}{ Depression } & Absent & Mild & Moderate & Moderately severe & Severe \\
\hline & $221(29 \%)$ & $324(43 \%)$ & $137(18 \%)$ & $52(7 \%)$ & $24(3 \%)$ \\
\hline \multirow[t]{2}{*}{ Anxiety } & Absent & Mild & Moderate & Severe & \\
\hline & $249(33 \%)$ & $350(46 \%)$ & $111(15 \%)$ & $48(6 \%)$ & \\
\hline \multirow[t]{2}{*}{ Anger } & Absent & Mild & Moderate & Severe & \\
\hline & $437(58 \%)$ & $116(15 \%)$ & $187(25 \%)$ & $18(2 \%)$ & \\
\hline
\end{tabular}

Frequency (percentage); $S D$ standard deviation

Bonferroni-corrected post hoc comparisons showed that PHQ-9 scores reported by young adults (mean $=7.98$; $\mathrm{SE}=0.26$ ) were significantly higher than those reported by middle-aged adults (mean $=6.45 ; \mathrm{SE}=0.32)$ and older adults $($ mean $=6.17 ; \mathrm{SE}=0.41)$ (corrected $-p=0.001$ for both); no significant difference was found on PHQ-9 score between middle-aged adults and older adults (corrected- $p=0.999$ ).

Neither the interactions between time and gender $\left(F_{(1,752)}=0.167 ; p=0.683 ; \eta_{p}^{2}=0.000\right]$, between time and $\operatorname{age}\left(\left[F_{(2,752)}=0.972 ; p=0.379 ; \eta_{p}^{2}=0.003\right)\right.$, and between gender and age $\left(F_{(2,752)}=0.949 ; p=0.387 ; \eta_{p}^{2}=0.003\right)$, nor the interaction among time, age, and gender $\left(F_{(2,752)}=1.831\right.$; $p=0.161 ; \eta_{p}^{2}=0.005$ ) was statistically significant.

\section{Anxiety}

As for anxiety, there was a significant main effect of the gender $\left(F_{(1,752)}=11.060 ; p=0.001 ; \eta_{p}^{2}=0.014\right)$ with females (mean $=6.77 ; \mathrm{SE}=0.17$ ) reporting a higher score on GAD-7 than males ( mean $=5.69 ; \mathrm{SE}=0.28$ ). Moreover, the analysis of anxiety symptoms showed a significant main effect of age $\left(F_{(2,752)}=6.472 ; p=0.002 ; \eta_{p}^{2}=0.017\right)$. Post hoc comparisons with Bonferroni's correction showed that young adults reported higher scores on GAD-7 (mean $=6.93$; $\mathrm{SE}=0.22)$ than middle-aged adults (mean $=5.75 ; \mathrm{SE}=0.27)$ (corrected- $p=0.002$ ); no significant differences on GAD-7 scores were found between young adults and older adults (corrected- $p=0.078$ ) and between middle-aged adults and older adults (corrected- $p=0.999$ ).

No significant main effect of the time $\left(F_{(1,752)}=0.674\right.$; $\left.p=0.412 ; \eta_{p}^{2}=0.001\right)$ on anxiety symptoms was found. Neither the interactions between time and gender $\left(F_{(1,752)}=0.111 ; p=0.739 ; \eta_{p}^{2}=0.000\right)$, between time and age $\left(F_{(2,752)}=1.160 ; p=0.314 ; \eta_{p}^{2}=0.003\right)$, and between gender and age $\left(F_{(2,752)}=1.448 ; p=0.236 ; \eta_{p}^{2}=0.004\right)$, nor the interaction among time, gender, and age $\left(F_{(2,752)}=1.468\right.$; $\left.p=0.231 ; \eta_{p}^{2}=0.004\right)$ was statistically significant.

\section{Anger}

Analysis of anger symptoms revealed no significant main effect of the time $\left(F_{(1,752)}=0.506 ; p=4.777 ; \eta_{p}^{2}=0.001\right)$, whereas there was a significant main effect of gender $\left(F_{(1,752)}=8.097 ; p=0.005 ; \eta_{p}^{2}=0.011\right)$ with females reporting higher scores on DSM-5-Anger (mean $=53.75$; $\mathrm{SE}=0.37)$ than males $($ mean $=51.73 ; \mathrm{SE}=0.61)$ (corrected- $p=0.005$ ). Moreover, we found a significant main effect of age $\left(F_{(2,752)}=10.283 ; p<0.001 ; \eta_{p}^{2}=0.027\right)$. Post-hoc comparisons with Bonferroni's correction showed that young adults reported higher scores on DSM-5-Anger $($ mean $=54.75 ; \mathrm{SE}=0.47)$ than middle-aged adults $($ mean $=52.42 ; \mathrm{SE}=0.59)$ and older adults $($ mean $=51.05$; $\mathrm{SE}=0.75$ ) (corrected- $p=0.006$ and corrected $-p<0.001$, respectively); no significant differences on DSM-5-Anger 
Fig. 1 Graph showing the interaction between time and gender for DSM-5-Anger scores

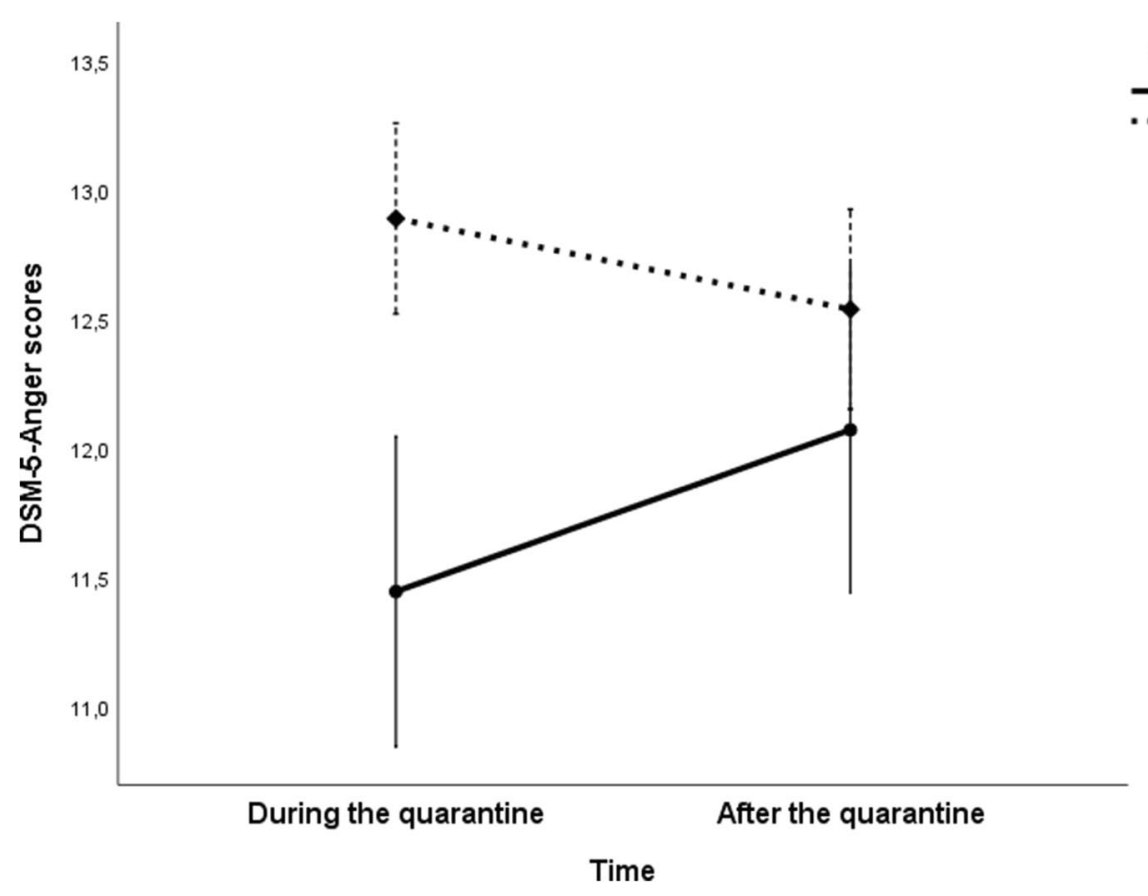

Gender

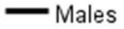
-. Females scores were found between middle-aged adults and older adults (corrected- $p=0.455$ ).

There was a significant interaction between time and gender $\left(F_{(1,752)}=7.557 ; p=0.006 ; \eta_{p}^{2}=0.010\right)$ (Fig. 1). Bonferroni-corrected post hoc comparisons showed that DSM-5-Anger scores were significantly higher in females ( mean $=54.16 ; \mathrm{SE}=0.42)$ than in males $($ mean $=51.03$; $\mathrm{SE}=0.68$ ), but only at baseline (corrected- $p<0.001$ ); no significant difference was found on anger symptoms between males and females at follow-up (corrected- $p=0.273$ ). Moreover, scores on DSM-5-Anger were significantly higher at follow-up (mean $=52.42 ; \mathrm{SD}=0.72)$ than baseline (mean $=51.03$; $\mathrm{SD}=0.68$ ) only in males (corrected$p=0.043$ ) but not in females (corrected- $p=0.052$ ).

Moreover, we found a significant interaction between time and age $\left(F_{(2,752)}=8.376 ; p<0.001 ; \eta_{p}^{2}=0.022\right)$ (Fig. 2). Post hoc comparisons with Bonferroni's correction revealed that DSM-5-Anger scores, at baseline, were significantly higher in young adults (mean $=55.56$; $\mathrm{SE}=0.53$ ) than in middle-aged adults (mean $=52.40$; $\mathrm{SE}=0.66)$ and older adults $($ mean $=49.83 ; \mathrm{SE}=0.84)$ (corrected- $p=0.001$ and corrected- $p<0.001$, respectively) and that middle-aged adults reported more severe anger symptoms than older adults (corrected- $p=0.049$ ); no significant differences was found on anger symptoms between young, middle-aged, and older adults at followup. Furthermore, anger scores significantly decreased at follow-up $($ mean $=53.94 ; \mathrm{SE}=0.56)$ compared to baseline $($ mean $=55.56 ; \mathrm{SE}=0.53)$ in young adults (corrected$p=0.002)$, whereas DSM-5-Anger scores increased from baseline $($ mean $=49.83 ; \mathrm{SE}=0.84)$ to follow-up
( mean $=52.26 ; \mathrm{SE}=0.89)$ in older adults (corrected$p=0.004)$; no significant difference was found between baseline and follow-up anger scores in middle-aged adults group (corrected- $p=0.950$ ).

No significant interaction between gender and age $\left(F_{(2,752)}=2.092 ; p=0.124 ; \eta_{p}^{2}=0.006\right)$, nor the interaction among time, gender, and age $\left(F_{(2,752)}=1.846 ; p=0.159\right.$; $\left.\eta_{p}^{2}=0.005\right)$ was found.

\section{Subjective cognitive failures}

Analysis of subjective cognitive failures showed a significant main effect of time $\left(F_{(1,752)}=23.988 ; p<0.001\right.$; $\left.\eta_{p}^{2}=0.031\right)$ with participants reporting higher PerMAFaQ scores at follow-up $($ mean $=18.23 ; \mathrm{SE}=0.31)$ rather than at baseline $($ mean $=17.03 ; \mathrm{SE}=0.28)$ (corrected$p<0.001$ ) (Fig. 3). Moreover, we found a significant main effect of gender $\left(F_{(1,752)}=15.113 ; p<0.001 ; \eta_{p}^{2}=\right.$ $0.020)$ with females reporting more severe subjective cognitive failures (mean $=18.67 ; \mathrm{SE}=0.28)$ than males $($ mean $=16.58 ; \mathrm{SE}=0.46)($ corrected $-p<0.001)$.

No significant main effect of age $\left(F_{(2,752)}=2.590\right.$; $\left.p=0.076 ; \eta_{p}^{2}=0.007\right]$ on PerMAFaQ scores was found. Neither the interactions between time and gender $\left(F_{(1,752)}=0.500 ; p=0.480 ; \eta_{p}^{2}=0.001\right)$, between time and age $\left(F_{(2,752)}=0.070 ; p=0.933 ; \eta_{p}^{2}=0.000\right)$, and between gender and age $\left(F_{(2,752)}=0.164 ; p=0.849 ; \eta_{p}^{2}=\right.$ $0.000)$, nor the interaction among time, gender, and age $\left(F_{(2,752)}=1.017 ; p=0.362 ; \eta_{p}^{2}=0.003\right)$ was statistically significant. 


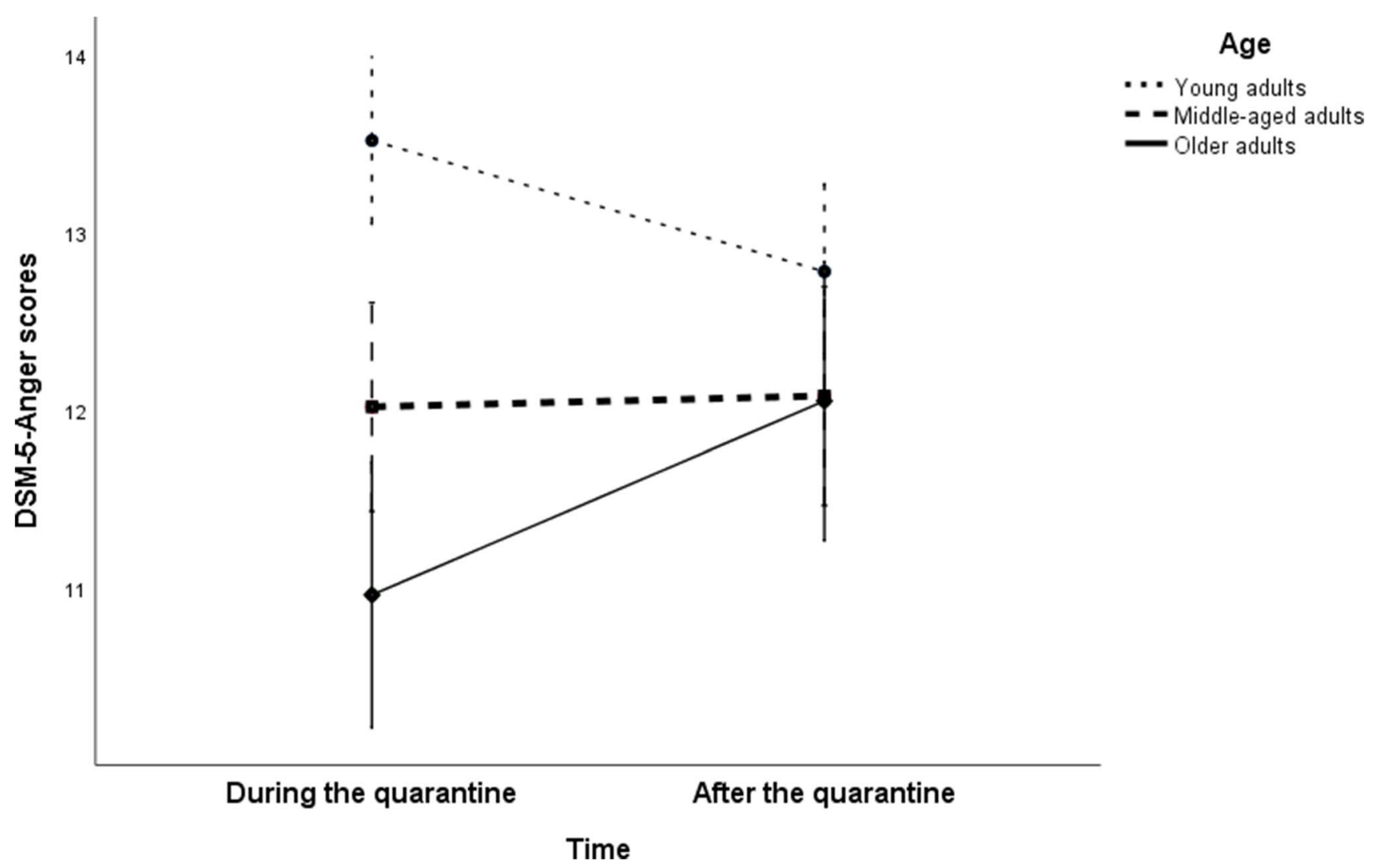

Fig. 2 Graph showing the interaction between time and age for DSM-5-Anger scores

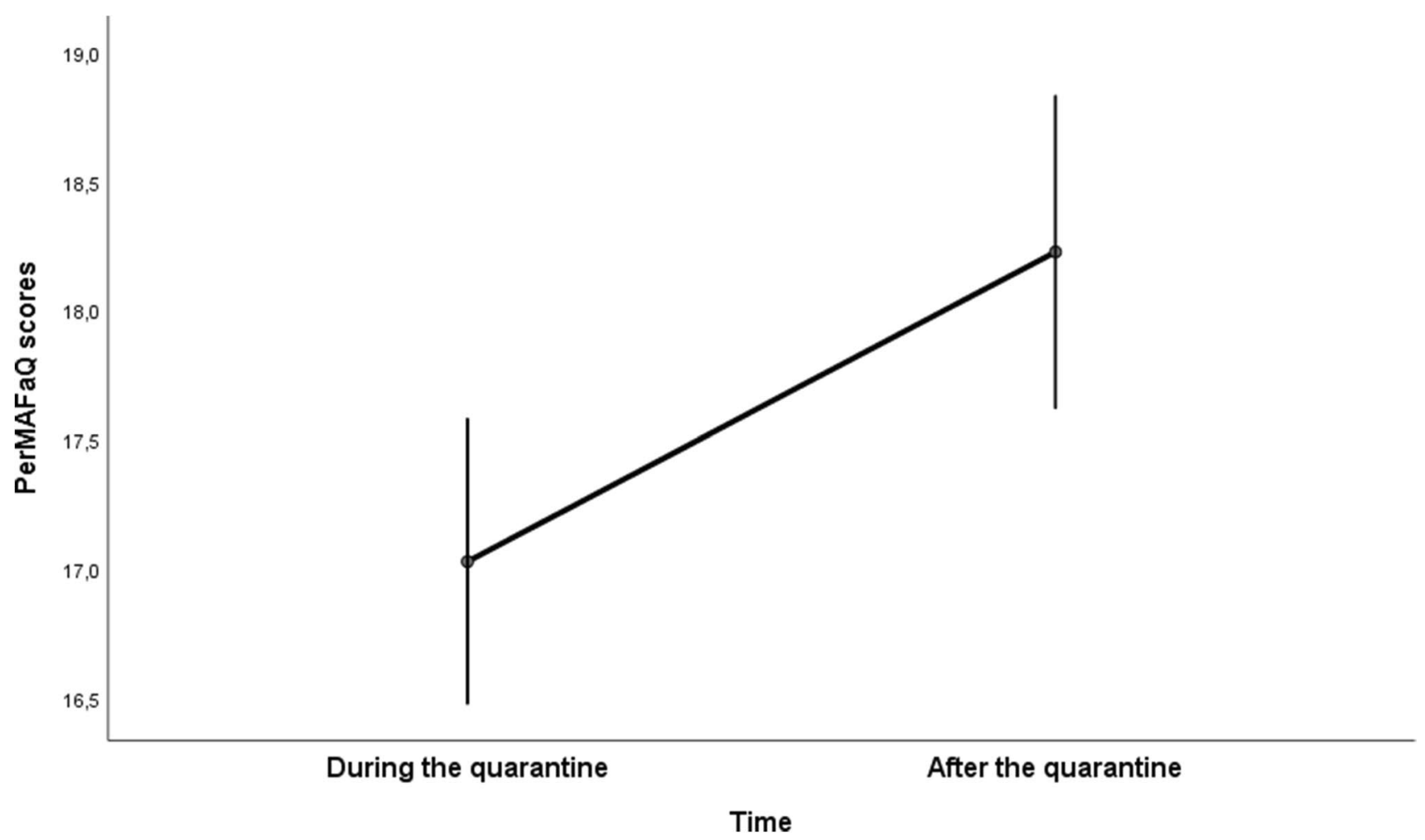

Fig. 3 Graph showing the main effect of time for PerMAFaQ scores 


\section{Resilience}

Analysis of resilience abilities revealed no significant main effect of time $\left(F_{(1,752)}=0.745 ; p=0.388 ; \eta_{p}^{2}=0.001\right)$, whereas there was a significant main effect of gender $\left(F_{(1,752)}=9.330 ; p=0.002 ; \eta_{p}^{2}=0.012\right)$ with males reporting more resilience $($ mean $=21.05 ; \mathrm{SE}=0.31)$ than females $($ mean $=19.92 ; \mathrm{SE}=0.19)$ (corrected- $p=0.002)$.

Moreover, we found a significant main effect of age $\left(F_{(2,752)}=15.871 ; p<0.001 ; \eta_{p}^{2}=0.041\right)$. Bonferronicorrected post hoc comparisons showed that BRS scores reported by young adults $($ mean $=19.27 ; \mathrm{SE}=0.24)$ were significantly lower than those reported by middleaged adults $($ mean $=21.38 ; \mathrm{SE}=0.31)$ and older adults (mean $=20.79 ; \mathrm{SE}=0.39$ ) (corrected- $p<0.001$ corrected$p=0.003$, respectively); no significant difference was found on BRS score between middle-aged adults and older adults (corrected- $p=0.696$ ).

Neither the interactions between time and gender $\left(F_{(1,752)}=0.873 ; p=0.350 ; \eta_{p}^{2}=0.001\right)$, between time and age $\left(F_{(2,752)}=0.487 ; p=0.615 ; \eta_{p}^{2}=0.001\right)$, and between gender and age $\left(F_{(2,752)}=0.637 ; p=0.529 ; \eta_{p}^{2}=0.002\right)$, nor the interaction among time, age, and gender $\left(F_{(2,752)}=0.245\right.$; $\left.p=0.782 ; \eta_{p}^{2}=0.001\right)$ was statistically significant.

\section{Coping}

We found a significant main effect of time $\left(F_{(1,752)}=6.521\right.$; $\left.p=0.011 ; \eta_{p}^{2}=0.009\right)$ on Coping Scale with participants reporting significantly lower scores (corrected- $p=0.011$ ) at follow-up (mean $=34.57 ; \mathrm{SE}=0.23$ ) compared to baseline (mean $=35.03 ; \mathrm{SE}=0.22)$; conversely, no significant main effect of age $\left(F_{(2,752)}=2.202 ; p=0.111 ; \eta_{p}^{2}=0.006\right)$ was found.

Moreover, we found a significant main effect of gen$\operatorname{der}\left(F_{(1,752)}=6.434 ; p=0.011 ; \eta_{p}^{2}=0.008\right)$ with males reporting higher scores on Coping Scale (mean $=35.31$; $\mathrm{SE}=0.35)$ than females $($ mean $=34.28 ; \mathrm{SE}=0.21)$ (corrected- $p=0.011$ ).

No significant interactions between time and gender $\left(F_{(1,752)}=0.376 ; p=0.540 ; \eta_{p}^{2}=0.001\right)$, between time and age $\left(F_{(2,752)}=0.185 ; p=0.831 ; \eta_{p}^{2}=0.000\right)$, and between gender and age $\left(F_{(2,752)}=0.101 ; p=0.904 ; \eta_{p}^{2}=0.000\right)$, nor significant interaction among time, age, and gender $\left(F_{(2,752)}=1.706 ; p=0.182 ; \eta_{p}^{2}=0.005\right)$ was found.

\section{Predictors of post-traumatic stress symptoms within the whole sample}

PTS symptoms, evaluated by IES-R, were subclinical or absent for $14.4 \%$ (109) of respondents, whereas they were mild for $55.8 \%$ (423), moderate for $23.5 \%$ (178), and severe for $6.3 \%$ (48). The mean score of IES-R was 20.73 (SD: 12.80).

Simple regression analyses revealed that the development of post-traumatic stress symptoms at follow-up was significantly related to female gender, less resilience, more fear of getting infected, more cognitive failures, more severe depressive, anxiety, and anger symptoms at baseline (Table 2). Multiple regression analysis showed that more severe PTS symptoms at follow-up were significantly related to more severe depressive and anxiety symptoms, more fear of getting infected, and more severe subjective cognitive failures at baseline (Table 2).

\section{Predictors of post-traumatic stress symptoms within male participants}

Considering male participants, PTS symptoms were subclinical or absent for $19.3 \%$ (37) of respondents, mild for $59.9 \%$ (115), moderate for $16.7 \%$ (32), and severe for $4.2 \%$ (8). The mean score of IES-R was 18.04 (SD: 12.35).

Simple regression analyses revealed that the development of post-traumatic stress symptoms at follow-up in males was significantly related to less resilience, more fear of getting infected, more severe cognitive failures, more severe depressive, anxiety, and anger symptoms at baseline (Supplemental Material 2). Multiple regression analysis showed that more severe PTS symptoms at follow-up in males were significantly related to more severe anxiety symptoms and more fear of getting infected at baseline (Supplemental Material 2).

\section{Predictors of post-traumatic stress symptoms within female participants}

In females, PTS symptoms were subclinical or absent for $12.7 \%$ (72) of respondents, mild for $54.4 \%$ (308), moderate for $25.8 \%$ (146), and severe for $7.1 \%$ (40). The mean score of IES-R was 21.64 (SD: 12.84).

Simple regression analyses revealed that the development of post-traumatic stress symptoms at followup in females was significantly related to lower educational level, less resilience, more fear of getting infected, more severe cognitive failures, more severe depressive, anxiety, and anger symptoms at baseline (Supplemental Material 3). Multiple regression analysis showed that more severe PTS symptoms at follow-up in females were significantly related to more severe anxiety and anger symptoms, more fear of getting infected, and more severe subjective cognitive failures at baseline (Supplemental Material 3). 
Table 2 Results for regression analyses with IES-R score computed as dependent variable within the whole sample

\begin{tabular}{|c|c|c|c|c|c|c|c|c|c|c|}
\hline & \multicolumn{3}{|c|}{ Simple regression analyses } & \multicolumn{2}{|c|}{$95 \%$ confidence limits } & \multicolumn{3}{|c|}{ Multiple regression analysis } & \multicolumn{2}{|c|}{$\begin{array}{l}95 \% \text { confidence } \\
\text { limits }\end{array}$} \\
\hline & Beta & $t$ & $p$ & Lower & Upper & Beta & $t$ & $p$ & Lower & Upper \\
\hline Age & -0.024 & -0.669 & 0.503 & -0.093 & 0.045 & & & & & \\
\hline Sex & 0.122 & 3.389 & 0.001 & 1.515 & 5.685 & - & - & - & - & - \\
\hline Education & -0.062 & -1.701 & 0.089 & -0.679 & 0.049 & & & & & \\
\hline Days of self-isolation & 0.037 & 1.021 & 0.308 & -0.040 & 0.127 & & & & & \\
\hline No. of people & 0.045 & 1.240 & 0.215 & -0.269 & 1.190 & & & & & \\
\hline No. of children & -0.052 & -1.440 & 0.150 & -2.382 & 0.366 & & & & & \\
\hline No. of rooms & 0.036 & 0.994 & 0.321 & -0.270 & 0.825 & & & & & \\
\hline No. of times leaving & -0.058 & -1.590 & 0.112 & -0.966 & 0.101 & & & & & \\
\hline Resilience & -0.263 & -7.485 & $<0.001$ & -0.918 & -0.536 & - & - & - & - & - \\
\hline Coping & -0.051 & -1.411 & 0.159 & -0.319 & 0.052 & & & & & \\
\hline Infected people & -0.065 & -1.209 & 0.228 & -0.408 & 0.097 & & & & & \\
\hline Fear & 0.275 & 7.867 & $<0.001$ & 2.665 & 4.437 & 0.149 & 4.536 & $<0.001$ & 1.095 & 2.765 \\
\hline PHQ-9 & 0.403 & 12.091 & $<0.001$ & 0.954 & 1.324 & 0.107 & 2.346 & 0.019 & 0.049 & 0.555 \\
\hline GAD-7 & 0.487 & 15.347 & $<0.001$ & 1.371 & 1.774 & 0.307 & 7.150 & $<0.001$ & 0.718 & 1.262 \\
\hline DSM-5-Anger & 0.389 & 11.617 & $<0.001$ & 0.447 & 0.629 & - & - & - & - & - \\
\hline PerMAFaQ & 0.364 & 10.730 & $<0.001$ & 0.600 & 0.868 & 0.144 & 3.729 & $<0.001$ & 0.137 & 0.443 \\
\hline
\end{tabular}

IES-R Impact of Event Scale-Revised, $P H Q-9$ Patient Health Questionnaire-9, GAD-7 7-item Generalized Anxiety Disorder scale, DSM-5-Anger DSM-5 Level 2-Anger-Adult measure, PerMAFaQ Perceived Memory and Attentional Failures Questionnaire

\section{Discussion}

In the present study, we explored the evolution of mental health status analyzing possible gender- and age-related differences and the predictors of the development of PTS symptoms in the Italian general population after the end of the quarantine due to COVID-19.

We found that younger individuals experienced more severe depressive symptoms than middle-aged and older people whereas no gender difference was found.

Previous studies demonstrated the adverse consequences of the COVID-19 pandemic in young adults who reported elevated depressive symptomatology [7]. Indeed, although young adults appear to be less vulnerable to the most serious medical complications associated with COVID-19, they experience psychological effects from the pandemic that can lead to risky behaviors such as alcohol use and suicide attempts $[7,8]$. This result can be due to the fact that self-restriction measures such as lockdown and self-isolation substantially limit young adults' everyday life activities heavily deflecting their mood.

The absence of a significant effect of gender on depressive symptoms appears to be an unusual result since the female gender has been often associated with a higher prevalence of depression during COVID-19 outbreak [18]. However, our findings are in line with those of a previous study on the Italian population [15] that revealed a slight improvement of depression in women rather than in men toward the end of the lockdown; thus, the gender gap detected in the first part of the lockdown was narrowed after 4 weeks.

Moreover, age- and gender-related differences were found on anxiety levels. Particularly, females reported higher anxiety scores than males and a significant difference was found in anxiety levels between young adults and middle-aged individuals.

A gender gap in the prevalence of anxiety and trauma and stress-related disorders was widely demonstrated in the psychiatric literature [19]. Particularly, Li and Graham [20] hypothesized that women might be more vulnerable to these disorders as a result of the monthly and/or life-span fluctuations of sex hormones such as estradiol and progesterone, with a significant impact on cognition and behavioral processes.

The findings of higher levels of anxiety levels in young adults rather than middle-aged individuals are in line with previous findings $[9,21]$ and could be due to several factors such as the uncertain working and financial conditions and the harsh limitations on their social activities. However, we did not observe any time effect on both depressive and anxiety symptoms. These results suggest that psychological consequences of the quarantine due to the COVID-19 pandemic persist also 2 months after the end of the lockdown highlighting the need to implement early psychological support interventions to avoid severe long-term consequences on psychological functioning. 
As for anger, we found a significant interaction between time and gender. More specifically, females reported more severe anger symptoms than males at baseline whereas no difference was found at follow-up evaluation. Moreover, males but not females reported an increase in anger scores at follow-up evaluation. These findings further support those provided by Salfi and colleagues [15] who reported how women coped better with the situation as the quarantine progressed whereas men showed a substantial increment of perceived stress at the end of lockdown.

The authors suggested that these results may be attributed to gender differences in stress response: men's first reaction to stress is generally a "fight-or-flight" reaction, whereas women's responses are more marked by a pattern of "tendand-befriend" [22]; thus, women's responses seem to be more appropriate in the current situation given the demonstrated importance of community support and prosocial behaviors in the COVID-19 pandemic [23]

A significant interaction on anger symptoms was found also between time and age. Particularly, we found that, at baseline, young adults experienced more severe anger symptoms than middle-aged adults, who in turn reported more severe anger than older adults, with the following order of severity: young adults $>$ middle-aged adults $>$ older adults. Furthermore, comparing baseline and follow-up scores, we observed a reduction of anger symptoms in young adults but an increase in older adults.

Therefore, the severity of anger symptoms at baseline was inversely proportional to age with young adults reporting higher scores than older individuals. These results further confirm the findings of a longitudinal study [24] that showed increased levels of anger in young adults during the pandemic compared to before.

We can assume that the severe restrictions imposed with lockdown, which often result in the inability to see the loved ones, in addition to changes in visions and hopes for their professional and economic future due to the economic crisis, may account for anger symptoms in young adults.

The abovementioned hypothesis was supported by the fact that we observed decreased anger levels after the end of the lockdown in young adults. Therefore, lockdown lifting and the partial return to everyday life improved anger symptoms in young adults. Conversely, a significant increase in anger scores was found in older adults at follow-up evaluation. Although anger and confrontation have been associated with younger age, a study conducted in the UK [25] revealed that anger symptoms seem to be related to a greater perceived risk of COVID-19. Indeed, older adults are more vulnerable to COVID-19 negative consequences and the fear of getting infected was associated with more severe anger symptoms [10]; thus, the increasing anger in older adults at follow-up might be due to a greater perceived risk of getting infected given the lack of restrictions in that phase of the pandemic.
However, anger is also caused by a higher reliance on social media as an information source about COVID-19 [25] but in turn, contributes to the spread of COVID-19 misinformation on social media [26]. A possible solution to stop this disagreeable loop can be to avoid excessive exposure to COVID-19 media coverage ensuring to be well-informed via reliable media sources.

Furthermore, we observed a significant main effect of time on subjective cognitive failures. More specifically, participants reported more severe cognitive failures at follow-up compared to baseline.

A recent review [27] reported how a paucity of social interaction and loneliness, as imposed to control the COVID19 pandemic, may have deleterious effects on the cognitive and memory systems. These results further confirm previous findings of more severe subjective cognitive deficits due to quarantine for COVID-19 in the Italian population [4, $10,28]$. To prevent long-term cognitive consequences, in a previous study [4], we suggested the need to implement psychoeducational interventions to foster resilience since it appears to limit the effects of mental disorders on cognition.

Indeed, resilience, rather than coping strategies, seems to be a protective factor in the development of mental health disorders in stressful situations such as quarantine due to the COVID-19 pandemic [4, 10, 29]. Moreover, we found no significant time effect on resilience whereas the adoption of coping strategies was lower at follow-up compared to baseline.

This confirms the idea that resilience is a stable trait that is not affected by adversity [30], whereas the adoption of coping strategies requires cognitive and behavioral efforts [31] that individuals cannot employ for a long time in prolonged stressful situations such as COVID-19 pandemic. Furthermore, the significant main effects of gender and age emerged analyzing resilience abilities and the significant main effect of gender observed on coping strategies further provide evidence on the idea that females and young adults seem to be more vulnerable groups.

Finally, about $30 \%$ of participants reported PTS symptoms 2 months after the end of the lockdown, and the development of these symptoms was associated with female gender, less resilience, more severe anger, more severe depressive and anxiety symptoms, more severe subjective cognitive failures, and more fear of getting infected measured during the quarantine as the most influential associated factors.

The result of more severe PTS symptoms at follow-up in females gender further supports previous results of more stress symptoms in females during the quarantine [16, 17].

Thus, we explored the predictors of PTS symptoms within male and female respondents, separately. We found that more severe anxiety and anger symptoms, more severe subjective cognitive failures, and more fear of getting infected 
were associated with PTS symptoms at follow-up in females, whereas more severe anxiety symptoms and more fear of getting infected were the significant predictors of PTS symptoms in males participants.

In a previous study [10], we revealed that the perceived vulnerability to disease plays a crucial role in the development of psychological symptoms in the elderly population but the fear seems to be also involved in the development of PTS symptoms in the general population. Moreover, 30\% of the Italian general population complained of cognitive failures during the quarantine for COVID-19 [4] and these, together with more severe psychological symptoms, represent risk factors for the development of PTS symptoms.

The result of more PTS symptoms at follow-up in female gender further supports previous results of more stress symptoms in females during the quarantine [16, 17].

Taking into account that the evolution from PTS symptoms in PTSD leads to significant physical and psychological impairments, we suggest developing early intervention programs to reduce the impact of traumatic events on individuals' mental health.

Although we analyzed only the responses provided by individuals who completed both the evaluations, the fact that only $18.16 \%$ of participants recruited during the first wave [4] completed the follow-up evaluation represents a limitation of the present study.

However, this drop-out rate is in line with other longitudinal studies conducted on the Italian population $[14,15]$ and could be due to the fact that people spent less time at home after the lockdown lifting and they were also less willing to complete a survey focused on an issue that appeared to be almost solved after the end of the first wave of the pandemic. Moreover, the differences in the age groups' size, probably due to the adopted convenience sampling methodology, could also limit our findings.

In conclusion, we explored, for the first time, psychological changes in the Italian population 2 months after the end of the lockdown. We observed no reduction in depressive and anxiety symptoms in the Italian general population compared to baseline with females and young adults reporting more severe psychological symptoms. Conversely, anger symptoms decreased in young adults whereas they increased in older adults; therefore, the end of the lockdown and selfrestriction measures produced different anger responses according to age. More severe subjective cognitive failures were observed at follow-up independently of age and gender suggesting that psychological symptoms could lead to long-term cognitive consequences. Moreover, our findings confirmed how resilience is a stable trait whereas adopting coping strategies could not be suitable in prolonged stressful situations. Finally, more severe psychological symptoms in addition to the fear of getting infected were associated with the development of PTS symptoms.
The present study indicates more vulnerable groups of subjects to mental health disorders who have to be identified in order to implement early interventions to reduce psychological symptoms avoiding the development of PTSD and cognitive disorders.

Supplementary Information The online version contains supplementary material available at https://doi.org/10.1007/s10072-021-05768-0.

Author contribution G.M. contributed substantially to the conception or design of the work, carried out the analysis and interpretation of the work's data, and wrote draft. I.B., A.B., N.D.C., M.C., and R.N. contributed substantially to the execution of the study and carried out the analysis and interpretation of the work's data. G.S. critically reviewed it for its important intellectual content.

All authors have approved the final article.

Availability of data and material Data and materials will be shared on request.

\section{Declarations}

Ethics approval The study was approved by the Local Ethics Committee and conformed to the principles embodied in the Declaration of Helsinki (1964).

Consent to participate Informed consent was obtained from all individual participants included in the study.

Informed consent Informed consent was obtained from all participants included in the study.

Conflict of interest The authors declare no competing interests.

\section{References}

1. Ilardi A, Chieffi S, Iavarone A, Ilardi CR (2021) SARS-CoV-2 in Italy: population density correlates with morbidity and mortality. Jpn J Infect Dis. https://doi.org/10.7883/yoken.JJID.2020.200

2. Torales J, O'Higgins M, Castaldelli-Maia JM, Ventriglio A (2020) The outbreak of COVID-19 coronavirus and its impact on global mental health. Int J Soc Psychiatry

3. Xiong J, Lipsitz O, Nasri F, et al (2020) Impact of COVID-19 pandemic on mental health in the general population: a systematic review. J Affect Disord

4. Santangelo G, Baldassarre I, Barbaro A et al (2021) Subjective cognitive failures and their psychological correlates in a large Italian sample during quarantine/self-isolation for COVID-19. Neurol Sci. https://doi.org/10.1007/s10072-021-05268-1

5. Forte G, Favieri F, Tambelli R, Casagrande M (2020) COVID-19 pandemic in the italian population: validation of a post-traumatic stress disorder questionnaire and prevalence of PTSD symptomatology. Int J Environ Res Public Health. https://doi.org/10.3390/ ijerph17114151

6. Luo M, Guo L, Yu M, Wang H (2020) The psychological and mental impact of coronavirus disease 2019 (COVID-19) on medical staff and general public - a systematic review and meta-analysis. Psychiatry Res

7. Charles NE, Strong SJ, Burns LC et al (2021) Increased mood disorder symptoms, perceived stress, and alcohol use among college 
students during the COVID-19 pandemic. Psychiatry Res. https:// doi.org/10.1016/j.psychres.2021.113706

8. Gracia R, Pamias M, Mortier P, et al (2021) Is the COVID-19 pandemic a risk factor for suicide attempts in adolescent girls? $\mathrm{J}$ Affect Disord

9. Pieh C, Budimir S, Probst T (2020) The effect of age, gender, income, work, and physical activity on mental health during coronavirus disease (COVID-19) lockdown in Austria. J Psychosom Res. https://doi.org/10.1016/j.jpsychores.2020.110186

10. Maggi G, Baldassarre I, Barbaro A et al (2021) Mental health status of Italian elderly subjects during and after quarantine for the COVID-19 pandemic: a cross-sectional and longitudinal study. Psychogeriatrics. https://doi.org/10.1111/psyg.12703

11. Meng H, Xu Y, Dai J, et al (2020) Analyze the psychological impact of COVID-19 among the elderly population in China and make corresponding suggestions. Psychiatry Res

12. Zhou T, Nguyen TVT, Zhong J, Liu J (2020) A COVID-19 descriptive study of life after lockdown in Wuhan, China: descriptive study of life after lockdown. R Soc Open Sci. https://doi.org/ 10.1098/rsos.200705

13. Daly M, Sutin A, Robinson E (2020) Longitudinal changes in mental health and the COVID-19 pandemic: evidence from the UK Household Longitudinal Study. Psychol Med. https://doi.org/ 10.1017/S0033291720004432

14. Roma P, Monaro M, Colasanti M et al (2020) A 2-month followup study of psychological distress among Italian people during the covid-19 lockdown. Int J Environ Res Public Health. https:// doi.org/10.3390/ijerph17218180

15. Salfi F, Lauriola M, Amicucci G et al (2020) Gender-related time course of sleep disturbances and psychological symptoms during the COVID-19 lockdown: a longitudinal study on the Italian population. Neurobiol Stress. https://doi.org/10.1016/j.ynstr.2020. 100259

16. Castelli L, Di Tella M, Benfante A, Romeo A (2020) The spread of COVID-19 in the Italian population: anxiety, depression, and post-traumatic stress symptoms. Can J Psychiatry 65:731-732. https://doi.org/10.1177/0706743720938598

17. Mazza C, Ricci E, Biondi S et al (2020) A nationwide survey of psychological distress among italian people during the covid-19 pandemic: immediate psychological responses and associated factors. Int J Environ Res Public Health. https://doi.org/10.3390/ijerp h17093165

18. Wang Y, Kala MP, Jafar TH (2020) Factors associated with psychological distress during the coronavirus disease 2019 (COVID19) pandemic on the predominantly general population: a systematic review and metaanalysis. PLoS One

19. Riecher-Rössler A (2017) Sex and gender differences in mental disorders. The Lancet Psychiatry
20. Li SH, Graham BM (2017) Why are women so vulnerable to anxiety, trauma-related and stress-related disorders? The potential role of sex hormones. Lancet Psychiatry

21. Huang Y, Zhao N (2020) Generalized anxiety disorder, depressive symptoms and sleep quality during COVID-19 outbreak in China: a web-based cross-sectional survey. Psychiatry Res. https://doi. org/10.1016/j.psychres.2020.112954

22. Taylor SE, Klein LC, Lewis BP et al (2000) Biobehavioral responses to stress in females: Tend-and-befriend, not fight-orflight. Psychol Rev. https://doi.org/10.1037/0033-295X.107.3.411

23. Van de Groep S, Zanolie K, Green KH et al (2020) A daily diary study on adolescents' mood, empathy, and prosocial behavior during the COVID-19 pandemic. PLoS ONE. https://doi.org/10.1371/ journal.pone. 0240349

24. Shanahan L, Steinhoff A, Bechtiger L et al (2020) Emotional distress in young adults during the COVID-19 pandemic: evidence of risk and resilience from a longitudinal cohort study. Psychol Med. https://doi.org/10.1017/S003329172000241X

25. Smith LE, Duffy B, Moxham-Hall V et al (2020) Anger and confrontation during the COVID-19 pandemic: a national crosssectional survey in the UK. J R Soc Med. https://doi.org/10.1177/ 0141076820962068

26. Han J, Cha M, Lee W (2020) Anger contributes to the spread of COVID-19 misinformation. Harvard Kennedy Sch Misinformation Rev. https://doi.org/10.37016/mr-2020-39

27. Bzdok D, Dunbar RIM (2020) The neurobiology of social distance. Trends Cogn Sci 24:717-733

28. Fiorenzato E, Zabberoni S, Costa A, Cona G (2021) Cognitive and mental health changes and their vulnerability factors related to COVID-19 lockdown in Italy. PLoS ONE 16:e0246204. https:// doi.org/10.1371/journal.pone.0246204

29. Kavčič T, Avsec A, Zager Kocjan G (2020) Psychological functioning of Slovene adults during the COVID-19 pandemic: does resilience matter? Psychiatr Q. https://doi.org/10.1007/ s11126-020-09789-4

30. Markovitz SE, Schrooten W, Arntz A, Peters ML (2015) Resilience as a predictor for emotional response to the diagnosis and surgery in breast cancer patients. Psychooncology. https://doi.org/ 10.1002/pon.3834

31. Lazarus RS, Folkman S (1984) Stress, appraisal, and coping Richard S. Lazarus, PhD, Susan Folkman, PhD

Publisher's note Springer Nature remains neutral with regard to jurisdictional claims in published maps and institutional affiliations. 